\title{
Clinical Application of Mathematical Long Bone Ratios to Calculate Appropriate Donor Limb Lengths in Bilateral Upper Limb Transplantation
}

\author{
Justyn Lutfy', Alexis Pietak'², Shaun D. Mendenhall', and Michael W. Neumeister'
}

\begin{abstract}
Background: Limited methods exist to aid in deciding the appropriate donor limb lengths in bilateral upper limb amputees qualifying for vascularized composite allotransplantation. We hypothesized mathematical equations could be created using long bone length ratios, and applied to radiographs, to approximate the patient's limb length prior to amputation. Methods: A data set of 30 skeletons' unilateral upper limb long bones measured using osteometric board and calipers was used. Anatomic segment ratios were calculated based on humerus length after multivariate linear regression analysis. For clinical application testing, 5 cadavers' upper limbs were radiographed. Radiographic bone lengths were then measured along the long axis of each long bone. These measured radiographic lengths were then compared with the predicted bone lengths, generated from the skeleton data set ratios, for each cadaver. Results: The chi-square goodness-of-fit test showed excellent fit $(P<.0 \mathrm{I})$ between the predicted and radiographically measured lengths for the 5 cadavers, and interobserver measurements showed no statistical difference. Depending on the cadaver, percent error in total limb length predicted to measure ranged from $0.9 \%$ to $2.7 \%$. The variables to multiply an individual humerus length to calculate a given anatomic segment thus proved to be effective. Conclusions: If a bilateral upper limb amputee has I intact humerus, ratios to the humerus length can be reliably applied to calculate the preamputation limb length based on the patient's radiographic humerus length. These formulas are indicated for finding the appropriate limb lengths, and smaller anatomic segments, for donor-recipient matching in upper limb transplantation.
\end{abstract}

Keywords: hand transplant, transplant limb length, arm transplant, vascularized composite allotransplantation, anatomic ratios

\section{Introduction}

What is the correct transplant limb length for a person with bilateral upper limb amputations? The answer may be hidden in Nature; we just need the math to figure it out.

There exists a Golden Ratio ( "1.618:1") in multiple natural elements as exemplified by the nautilus shell. The presence of the Golden Ratio in the proportions of the ideal human body has also been described since antiquity as exemplified by Leonardo da Vinci Vitruvian man. Today, the concept of the Golden Ratio defining facial "beauty" is well known in cosmetic surgery., ${ }^{3,13,17,23}$ Anatomic ratios are utilized in the art of palm reading with various finger-topalm-to-hand ratios to infer a person's traits such as ethnicity, character, and medical health. Scientific publications are showing that finger length ratios are influenced by fetal androgen exposure and do indeed relate to character and medical health. ${ }^{1,5,6,11,14,18,19,22,24,28}$ Anthropologic and forensic scientists use isolated long bone lengths to estimate the height of a person of an incomplete skeleton.

The practice of hand surgery gives little attention to the hand's anatomic ratios. Hutchison et al studied the landmark papers by Dr William Littler and other authors on the mathematical relationships within the hand. ${ }^{12}$ They found

\footnotetext{
'Southern Illinois University School of Medicine, Springfield, USA

${ }^{2}$ Tufts University, Medford, MA, USA

Supplemental material is available in the online version of the article.

Corresponding Author:

Justyn Lutfy, The Institute for Plastic Surgery, Southern Illinois University School of Medicine, 747 North Rutledge Street, \#3, Springfield, IL 62702, USA.

Email: justyn.lutfy@hotmail.com
} 
that the small finger's phalanges followed the Fibonacci series (and thus Golden Ratio), but that the index, long, and ring finger phalanges follow a ratio of $x, 1.3 x$, and $2.6 x$, where " $x$ " is the length of the distal phalanx. This ratio was coined as the "Littler series" by the authors.

With such strong evidence of constant ratios within nature's constructs, the first part of the study tested our hypothesis that conserved mathematical relationships exist between long bones of the upper limb with low variability between individuals. Thus, knowing the length of 1 bone segment within a limb would allow missing segments to be calculated. The second part of the study was with 5 cadavers to test whether the mathematical model would be potentially applicable in the clinical setting with bone lengths measured on radiographs. We therefore ultimately aim to determine a mathematical model that could calculate the missing anatomic segments and total limb length in candidates for bilateral upper limb transplantation when only the humerus length is known.

\section{Materials and Methods}

Statistical analysis was completed in the software package, $\mathrm{R}$, available from www.r-project.org. A value of $P<.05$ was taken as the level of statistical significance for all statistical tests.

The steps to achieve our goal were to first acquire skeletal long bone measurements, to calculate the mathematical relationships between bones, then acquire bone length measurements using clinical tools (radiographs and PACS viewer software), and, finally, to test the validity of the mathematical model on predicting other bone segments from the radiographically measured humerus.

\section{Section A}

Skeletal bone measurements. A population of skeletons was first required to perform the upper limb long bone measurements on and subsequent analysis. The raw data acquired by Pietak et al were sourced and used. ${ }^{21}$ In brief, a collection of 30 adult skeletons' unilateral upper limb long bones housed at the anatomy department of Otago University (Dunedin, New Zealand) were used. There were 19 males' and 11 females' upper limbs composed of Asian Indian or white European races, and half of the limbs were the right side. Measurements of the third ray phalanges and metacarpal, forearm bones, and humerus, excluding the carpal bones, were performed using an osteometric board or calipers. Thus, these measurements were the longest lengths parallel to the long axis of each bone from the most proximal to distal anatomic points.

The following acronyms are used to refer to segments of the limb: $\mathrm{H}=$ humerus; $\mathrm{RU}=$ radius/ulna average; $\mathrm{MC}=$ metacarpal (carpals excluded); $\mathrm{MC}+\mathrm{C}=$ metacarpal + estimated carpal height; $\mathrm{PP}=$ proximal phalanges; $\mathrm{MP}=$ middle phalanges; $\mathrm{DP}=$ distal phalanges.

As carpal bones were not measured in the Pietak et al study, we assumed an ideal carpal height ratio of 0.54 existed in each skeletal set and calculated the carpal height along the capitate and lunate. ${ }^{8}$ By adding together the metacarpal length $(\mathrm{MC})$ and carpal height $(\mathrm{C})$, a total palm height $(\mathrm{MC}+\mathrm{C})$ was found.

Creation of normalized limb ratios based on the humerus length. Using the bone lengths from Pietak et al study, we created sequences of bone ratios for each skeleton's humerus length to normalize the length of its other long bones lengths to. Multiple analysis of variance (MANOVA) was used to look for statistically significant differences in humerus-normalized arm segment ratios with gender, ethnicity (Asian/ Indian or Caucasian), and side (right or left) used as independent variables. As MANOVA indicated both gender and ethnicity to be independent variables with a significant impact on humerus-normalized arm segment ratio values, a complete regression model was constructed using a multiple multivariate linear regression analysis on the humerus-normalized segment sequences with both gender and ethnicity used as independent variables (see Supplementary Table S2). In addition to standard least squares regression model, a Least Absolute Shrinkage and Selection Operator (LASSO) was used to create a regression model allowing independent variables with a minor effect on the response to become zero. As the LASSO regression analysis indicated no benefit to inclusion of gender and ethnicity independent variables in the model, and the predictive capacity of the LASSO regression parameters was found to be superior to those from the complete regression model (see the "Results" section), we used the LASSO regression model parameters as the final humerus-normalized arm segment ratio values. We have called these resulting ratios the "Lutfy-Pietak" ratios (see "Section A" in the "Results" section and Table 1).

\section{Section $B$}

Measuring long bone lengths using clinical tools. To recreate the clinical setting of measuring long bone lengths, 5 fresh semipreserved adult cadavers' right upper extremities were radiographed. Four were female, 1 was male. Standard radiographs were taken of the third digit, hand, wrist, forearm, and humerus with a ruler in the image to confirm standard length measurements. Radiographs were examined for bony pathologies or significant arthropathies to exclude the cadaver limb from the study. Best visualization of long-axis bone length was on either the posterior-anterior (PA) or anterior-posterior (AP) views, and thus used for measurements.

As the initial measurements of the long bones were performed on an osteometric board, this was re-created within our center's PACS viewer (Centricity Universal Viewer, GE 
Table I. Values Used to Multiply a Radiographically Measured Humerus Length to Acquire the Missing Anatomic Segment Lengths of the Arm.

\begin{tabular}{lll}
\hline Segment & Ratio & \multicolumn{1}{c}{ SE } \\
\hline $\mathrm{RU} / \mathrm{H}$ & 0.79 & 0.04 \\
$\mathrm{MC} / \mathrm{H}$ & 0.22 & 0.01 \\
$(\mathrm{MC}+\mathrm{C}) / \mathrm{H}$ & 0.33 & 0.02 \\
$\mathrm{PP} / \mathrm{H}$ & 0.14 & 0.010 \\
$\mathrm{MP} / \mathrm{H}$ & 0.094 & 0.009 \\
$\mathrm{DP} / \mathrm{H}$ & 0.062 & 0.007 \\
\hline
\end{tabular}

Note. $\mathrm{RU} / \mathrm{H}=$ forearm scaled by humerus; $\mathrm{MC} / \mathrm{H}=$ third metacarpal scaled by humerus; $(\mathrm{MC}+\mathrm{C}) / \mathrm{H}=$ third metacarpal and carpus (ie, palm height) scaled by humerus; $\mathrm{PP} / \mathrm{H}=$ proximal phalanx long finger scaled by humerus; $\mathrm{MP} / \mathrm{H}=$ middle phalanx long finger scaled by humerus; $\mathrm{DP} / \mathrm{H}=$ distal phalanx long finger scaled by humerus.

Healthcare, Chicago, Illinois) using the "multi-Cobb angle" function. An "H" was created with the horizontal limb of the " $\mathrm{H}$ " in line with the axis of the long bone, and the vertical limbs corresponded to the most proximal and distal anatomic points of the bone (Figure 1). The length measuring tool was then used to measure the horizontal length of the " $\mathrm{H}$ " of all bones. The most proximal to distal points in each anatomic segment are as follows: humerus - head to trochlea, forearm - olecranon to radial styloid, carpal heightlunate fossa on radius to distal surface of capitates, third metacarpal — base to head, third digit phalanges - base to head. Two authors independently measured the anatomic segments, and the Bland-Altman method for interobserver variability was performed. ${ }^{16}$

To calculate total limb length, we used the equation: humerus + forearm + palm (carpal height + third metacarpal) + long finger phalanges $-1.2 \mathrm{~cm}$. The " $-1.2 \mathrm{~cm}$ " is derived from the accepted average radial height (sigmoid notch to radial styloid) to account for the overlap of the carpal bones and distal radius. ${ }^{7}$ Simply taking the sum of each anatomic segment would give a limb length equivalent to the carpus sitting on top of the radial styloid instead of resting within the lunate and scaphoid fossas. The overlap of the capitates and third metacarpal was negligible and excluded.

Predicting clinical cadaver bone lengths using the Lutfy-Pietak ratios. Last, testing the validity of the Lutfy-Pietak ratios in predicting long bone lengths based on radiograph humerus length was performed. Having acquired the radiographically measured long bone lengths of each cadaver, we applied the Lutfy-Pietak ratio set to the humerus length to obtain estimates of each remaining segment length in the $\mathrm{arm}$. We then tested the correspondence between the radiograph measured and Lutfy-Pietak ratio predicted segment lengths using the chi-square goodness-of-fit test. Using this approach, each cadaver acts as its own control because if the humerus length is measured incorrectly, all predicted long bone lengths would be incorrect, and there would be no consistency between the measured and predicted long bone lengths.

Discussion with our Institutional Review Board concluded that Ethics Board approval was not necessary for this cadaveric study.

\section{Results}

\section{Section A}

Creation of normalized limb ratios based on the humerus length. The humerus-normalized segment lengths showed conservation of the values of the resulting ratios between different individuals. Multivariate analysis of variance found both gender and race to have significant impacts on the ratio values (Supplemental Table S1). Therefore, a multiple multivariate linear regression analysis with gender and ethnicity was performed, which allowed corrections for gender and race to be applied to the humerus-normalized arm segment ratios (Supplemental Table S2). However, a LASSO regression indicated minimum error resulted for a regression model without gender and ethnicity descriptors, and in practice, the noncorrected, average ratios from the training data set produced the most accurate predictions. Thus, uncorrected humerus-normalized ratio sequences were chosen and are referred to as the "Lutfy-Pietak ratios" of Table 1.

\section{Section $B$}

Measuring long bone lengths using clinical tools. The BlandAltman analysis of the data shows high interobserver reliability in measured radiograph bone lengths, with nearly all measures falling within the $95 \%$ confidence interval (CI) bounds. The Bland-Altman plot for the total sum of measured upper limb segments (expected to contain the highest variability due to sum of compounded measures) for the 2 different observers is shown in Figure 2.

As found in the skeletal reference data set, the measurements of each anatomic segment were generally longer in the male, but the ratio of bone lengths to each other was consistent. Table 2 depicts the average measured lengths for each cadaver, along with predicted lengths and percent error between the radiograph measured and ratio predicted arm segments for each cadaver.

Chi-square goodness-of-fit analysis of predicted versus measured anatomic lengths, and total limb length, showed excellent fit for each cadaver $(P<.01)$. Furthermore, depending on the cadaver, total percent error of limb length varied between $0.1 \%$ and $2.7 \%$, corresponding to a 0.7 - to $2.3-\mathrm{cm}$ absolute length discrepancy range on the averaged cadaver limb length of $77 \mathrm{~cm}$. 

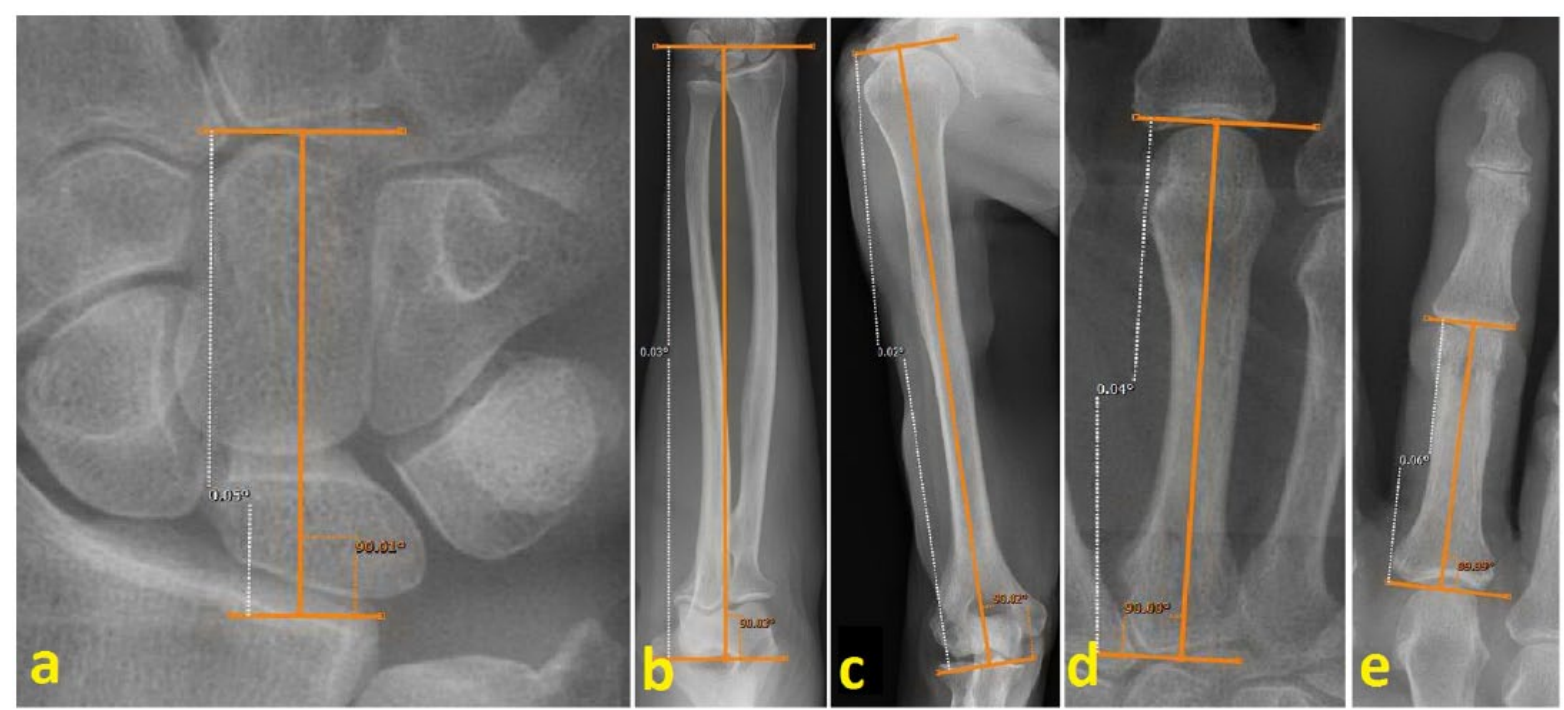

Figure I. Height measurements of (a) carpal height in posterior-anterior (PA), (b) forearm PA, (c) humerus anterior-posterior, (d) metacarpal PA, and (e) phalanx PA.

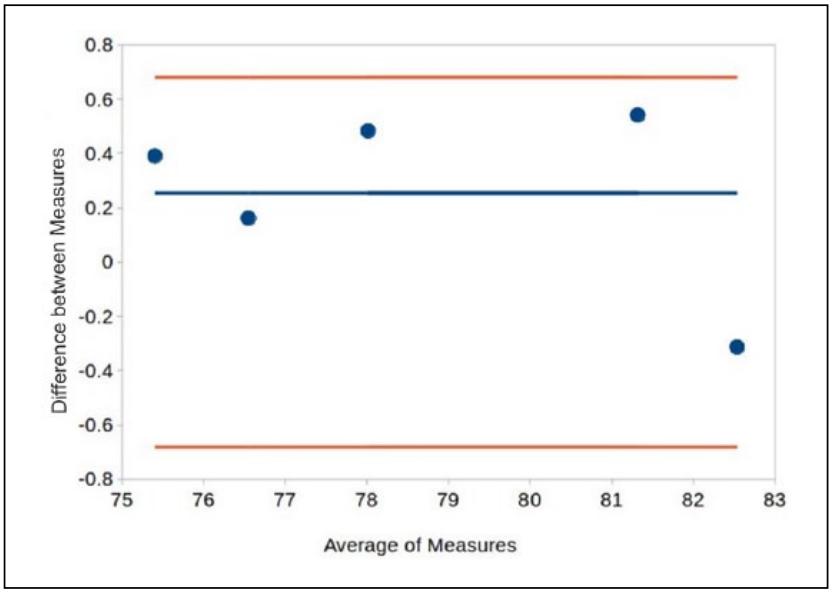

Figure 2. Bland-Altman plot for sum of limb segment measures recorded by the 2 study physicians.

Note. Red lines show the $95 \%$ confidence interval bounds for acceptable interobserver variability, whereas the blue line shows the mean of the observer value differences.

Thus, the Lutfy-Pietak ratios can be used on a radiographically measured humerus to reliably calculate the missing anatomic segments in bilateral upper limb amputees. For total limb length calculation from olecranon to the long finger tip, the following equation is used: $(1.4 \times$ humerus length $\mathrm{cm})-1.2 \mathrm{~cm}$.

\section{Discussion}

Inspired by the long bone ratios reported by other studies, we applied the concept to show that total upper limb length, and that of the anatomic subunits, can be reliably calculated based off of the radiographic humerus length and applying the Lutfy-Pietak ratios. The clinical application of this finding is important for the vascularized composite allotransplant community as it addresses the conundrum of deciding what the appropriate donor limb lengths should be for any given bilateral upper limb transplant candidate. The importance of this can be seen in the months after transplantation as poor proprioception, and noxious stimuli sensation lends itself to injury. However, if the transplanted limbs' lengths are closer to the brain's habituated spatial relationships, the requirement of visual input and cortical retraining may be lessened. ${ }^{9,26,27}$

The underlying consistency of the Lutfy-Pietak ratios likely relates to embryologic development of vertebrate animal limbs with morphogens interacting with one another between the apical ectodermal ridge, progress zone, and zone of polarizing activity to govern the final three-dimensional (3-D) development of the limb. Limb length is controlled by the apical ectodermal ridge and predominantly fibroblast growth factor (FGF)-driven. ${ }^{23,25}$ Multiple studies have shown through both biological and computer model predictions that a gradient exists down the limb in the expression of morphogens and this in turn controls segment size and thus overall length., ${ }^{2,23}$

Although one would assume that with the existence of an embryologic morphogen gradient driving limb segment lengths, that authors' conclusions of human hand biomechanics should all be the same, but they are not. Dr Littler's theory that hand phalanges' lengths follow the Golden Ratio ${ }^{15}$ has been studied previously with agreement and refute. This discrepancy arises from the 


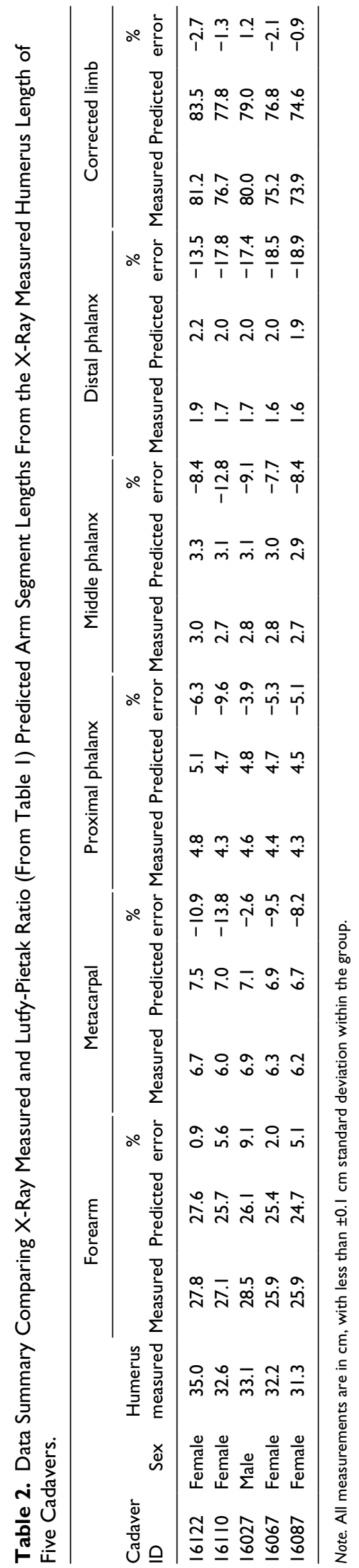


Table 3. Values to Multiply a Radiographically Measured Femur Length to Acquire the Missing Anatomic Segment Lengths of the Arm Based on Race (Caucasians or Asian/Indian), or a Combined Model.

\begin{tabular}{lccc}
\hline Segment & Caucasians & Asian/Indian & Combined \\
\hline RU / F & 0.54 & 0.57 & 0.56 \\
MC / F & 0.15 & 0.16 & 0.154 \\
$(\mathrm{MC}+\mathrm{C})$ / F & 0.23 & 0.24 & 0.24 \\
$\mathrm{PP} / \mathrm{F}$ & 0.10 & 0.10 & 0.103 \\
$\mathrm{MP} / \mathrm{F}$ & 0.069 & 0.067 & 0.067 \\
$\mathrm{DP} / \mathrm{F}$ & 0.05 & 0.04 & 0.044 \\
\hline
\end{tabular}

Note. $\mathrm{RU} / \mathrm{F}=$ forearm scaled by femur; $\mathrm{MC} / \mathrm{F}=$ third metacarpal scaled by femur; $(M C+C) / F=$ third metacarpal and carpus (ie, palm height) scaled by femur; $\mathrm{PP} / \mathrm{F}=$ proximal phalanx long finger scaled by femur; $\mathrm{MP} / \mathrm{F}=$ middle phalanx long finger scaled by femur; $D P / F=$ distal phalanx long finger scaled by femur.

various methods used to measure long bone length such as mid-axis proximal to distal articular surfaces, ${ }^{2}$ absolute bone length using osteometric board-like measurement, ${ }^{4,20}$ or axis of rotation of the phalanges. ${ }^{10}$ It was not until Hutchison and Hutchison reviewed the literature that it was noted that there likely exists 2 different Lucas ratios. ${ }^{11}$ Hutchison and Hutchison found that the small finger best approximated the Fibonacci sequence, but that the index, long, and ring fingers followed the Littler series. However, the studies used by Hutchison and Hutchison for their conclusion only reported ratios based off of averages, and the 2 studies quoted had different methods of length measuring. ${ }^{10,20}$ Out of curiosity, we pooled 30 skeleton's phalange lengths from the Pietak et al study. ${ }^{21}$ We found a Lucas series of $x, 1.5 x$, and $2.3 x$ was found for the relative phalanges lengths (unpublished data) and does not correspond to the "Littler series" of $x$, $1.3 x$, and $2.6 x$, and is likely due to different measuring technique. Still, the concept of maintained ratios was pushed further by Aydinlioglu et al who showed that constant ratios also exist across adjacent ray anatomic subunits ${ }^{2}$ and by Pietak et al in a logarithmic length relationship existing among the long bones of the limbs. ${ }^{21}$ Our study looked at long bone ratios in a novel way by using the humerus normalized to that person and showing the potential clinical utility of such ratios for upper limb transplantation.

The applicability of the Lutfy-Pietak ratios also extends to reconstructive surgery of the upper limbs. For example, in situations where only $1 \mathrm{limb}$ is salvageable and contains a segmental bony defect, the Lutfy-Pietak ratios can be used to achieve appropriate bone graft/flap lengths to help restore limb length, soft tissue harmony, and muscle function through appropriate length tensioning. Furthermore, we also believe that the Lutfy-Pietak ratios can be applied to custom-made, patient-specific bilateral upper limb prosthesis length calculations.
This study does have a few limitations. As the initial skeletal data set only measured long bones, we had to assume that each skeleton had a carpal height of 0.54 and radial height of $1.2 \mathrm{~cm}$, which are accepted anatomic norms, in creating our ratios and limb length equations. Only 5 cadavers were used in the clinical application of the ratios as this was the absolute limit of cadavers available to this study. Furthermore, on examination of the negative percent errors in Tables 2 and Supplemental Table S3, the ratios do have a tendency to overestimate the lengths. This is likely attributed to a systemic difference in measuring bone lengths between radiograph and osteometric board. Thus, a future study would aim to construct a new training set of date based solely on radiograph measurements to produce the most clinically applicable ratios.

While the findings in this study help in determining total limb lengths, matching of donor-recipient anatomic subunit will likely not be possible given the more important skin color and soft tissue bulk matching. Surgically, the importance of proper musculotendinous tensioning and tensionfree nerve coaptations is ever present. However, as immunosuppressant treatment advances, anatomic subunit lengths may become important if finger/ray transplantation becomes standard of care. The applicability of the study's findings is confined to individuals with normal limb anatomy and likely excludes those with generalized skeletal anomalies such as skeletal dysplasia, Marfan syndrome, or conditions relating to embryologic longitudinal arrest or hypoplasia/hyperplasia of the limbs.

Our group was hoping to complete the second study to examine other long bones for transplant candidates with bilateral humerus amputations or quadruple limb amputations. Using the ipsilateral femur from each of the 30 skeletons from the Pietak et al study, we were able to find another set of ratios between it and all the long bones of upper arm (Table 3). Using MANOVA, statistically significant differences were observed between ethnicity and ratios for individual's bone lengths when scaled by individual's femur length (Supplemental Table S1). As we are unable to complete the second study, using measurements of cadaver radiographs of femur and upper limb bones, we invite other investigators to test the reliability of these femur-derived Lutfy-Pietak ratios in calculating upper limb (segment) lengths. We found that total limb length from olecranon to the long finger tip can be calculated from the femur using the following equation: $(1.0 \times$ femur length $\mathrm{cm})-1.2 \mathrm{~cm}$.

\section{Conclusion}

This study presents mathematical ratios for calculating the appropriate donor limb lengths in bilateral upper limb vascularized composite allotransplantation candidates. This is accomplished using AP radiographs of the humerus and performing osteometric board-like measurements using standard PACS viewer tools. 
Further work is required to determine the precise value of ratios applicable to a wider population, and confirm whether race and age are indeed not variables and how syndromes may affect these ratios.

\section{Ethical Approval}

Discussion with our Institutional Review Board concluded that Ethics Board approval was not necessary for this cadaveric study.

\section{Statement of Human and Animal Rights}

Procedures followed were in accordance with the ethical standards of the responsible committee on human experimentation (institutional and national) and with the Helsinki Declaration of 1975, as revised in 2000 and 2008.

\section{Statement of Informed Consent}

Discussion with our Institutional Review Board concluded that Statement of Informed Consent was not applicable for this cadaveric study.

\section{Declaration of Conflicting Interests}

The author(s) declared no potential conflicts of interest with respect to the research, authorship, and/or publication of this article.

\section{Funding}

The author(s) disclosed receipt of the following financial support for the research, authorship, and/or publication of this article: This work was supported by a grant from the Memorial Medical Center Foundation.

\section{References}

1. Al-Zaid FS, Alhader AA, Al-Ayadhi LY. The second to fourth digit ratio (2D:4D) in Saudi boys with autism: a potential screening tool. Early Hum Dev. 2015;91(7):413-415.

2. Aydinlioglu A, Akpinar F, Tosun N. Mathematical relations between the lengths of the metacarpal bones and phalanges: surgical significance. Tohoku J Exp Med. 1998;185(3):209-216.

3. Bashour M. An objective system for measuring facial attractiveness. Plast Reconstr Surg. 2006;118(3):757-774; discussion $775-776$.

4. Buryanov A, Kotiuk V. Proportions of hand segments. Int $J$ Morphol. 2010;28:755-758.

5. Choi IH, Kim KH, Jung H, et al. Second to fourth digit ratio: a predictor of adult penile length. Asian J Androl. 2011;13(5):710-714.

6. de Bruin EI, Verheij F, Wiegman T, et al. Differences in finger length ratio between males with autism, pervasive developmental disorder-not otherwise specified, ADHD, and anxiety disorders. Dev Med Child Neuro. 2006;48(12):962-965.

7. Fernandez DL, Wolfe SW. Distal radius fractures. In: Green DP, editor in chief. Green's Operative Hand Surgery. 5th ed. Philadelphia, PA: Elsevier; 2005:645-710.

8. Garcias-Elias M. Carpal instability. In: Wolfe SW, editor in chief. Green's Operative Hand Surgery. 6th ed. Philadelphia, PA: Elsevier; 2011:465-522.
9. Gentilucci M, Toni I, Chieffi S, et al. The role of proprioception in the control of prehension movements: a kinematic study in a peripherally deafferented patient and in normal subjects. Exp Brain Res. 1994;99:483-500.

10. Hamilton R, Dunsmuir RA. Radiographic assessment of the relative lengths of the bones of the fingers of the human hand. J Hand Surg Br. 2002;27(6):546-548.

11. Hussain SM, Wang Y, Muller DC, et al. Association between index-to-ring finger length ratio and risk of severe knee and hip osteoarthritis requiring total joint replacement. Rheumatology (Oxford). 2014;53(7):1200-1207.

12. Hutchison AL, Hutchison RL. Fibonacci, littler, and the hand: a brief review. Hand (N Y). 2010;5(4):364-368.

13. Kim YH. Easy facial analysis using the facial golden mask. $J$ Craniofac Surg. 2007;18(3):643-649.

14. Lippa RA. Are 2D:4D finger-length ratios related to sexual orientation? yes for men, no for women. J Pers Soc Psychol. 2003;85(1):179-188.

15. Littler JW. On the adaptability of man's hand (with reference to the equiangular curve). Hand. 1973;5(3):187-191.

16. Martin Bland J, Altman D. Statistical methods for assessing agreement between two methods of clinical measurement. Lancet. 1986;327(8476):307-310.

17. Moskowitz DS, Sutton R, Zuroff DC, et al. Fetal exposure to androgens, as indicated by digit ratios (2D:4D), increases men's agreeableness with women. Pers Individ Differ. 2015;75:97101.

18. Moufarrège R. The golden ratios of the breast. In: Shiffman AM, ed. Breast Augmentation: Principles and Practice. Berlin, Germany: Springer; 2009:109-116.

19. Nikbakht N, McLachlan JC. A proximo-distal gradient of FGF-like activity in the embryonic chick limb bud. Cell Mol Life Sci. 1997;53(5):447-451.

20. Park AE, Fernadez JJ, Schmedders K, et al. The Fibonacci sequence: relationship to the human hand. J Hand Surg Am. 2003;28(1):157-160.

21. Pietak A, Ma S, Beck SW, et al. Fundamental ratios and logarithmic periodicity in human limb bones. J Anat. 2013;222(5):526-537.

22. Rahman AA, Lopthatananon A, Stewart-Brown S, et al. Hand pattern indicates prostate cancer risk. Br J Cancer. 2011;104(1):175-177.

23. Raspopovic J, Marcon L, Russo L, et al. Digit patterning is controlled by a Bmp-Sox9-Wnt Turing network modulated by morphogen gradients. Science. 2014;345(6196):566-570.

24. Swift A, Remington K. BeautiPHIcation ${ }^{\mathrm{TM}}$ : a global approach to facial beauty. Clin Plast Surg. 2011;38(3):347-377.v.

25. Tickle C. Morphogen gradients in vertebrate limb development. Semin Cell Dev Biol. 1999;10(3):345-351.

26. Vargas CD, Aballéa A, Rodrigues EC, et al. Re-emergence of hand-muscle representations in human motor cortex after hand allograft. Proc Natl Acad Sci U S A. 2009;106(17):7197-7202.

27. Wheaton LA. Neurorehabilitation in the upper limb amputation: understanding how neurophysiologic changes can affect functional rehabilitation. J Neuroeng Rehabil. 2017;14:41.

28. Wu XL, Yang DY, Chai WH, et al. The ratio of second to fourth digit length (2D:4D) and coronary artery disease in a Han Chinese population. Int J Med Sci. 2013;10(11):15841588. 